\title{
A Switched-Capacitor Successive-Approximation A/D Converter
}

\author{
Satomi Ogawa, Student Member, IEEE, and Kenzo Watanabe, Fellow, IEEE
}

\begin{abstract}
A switched-capacitor successive-approximation analog-to-digital (A/D) converter is developed which incorporates a serial digital-to-analog (D/A) subconverter for generating the threshold voltage sequence. The conversion process is insensitive to parasitic capacitances and offset voltages of a comparator and op-amps. Error analyses and Spice simulations show that a resolution higher than $11 \mathrm{~b}$, a sampling rate up to $440 \mathrm{ksps}$ with 10-b resolution, and a power consumption less than $60 \mathrm{~mW}$ are attainable with the monolithic implementation using present CMOS technologies. The required chip area is small because of a low device count. The architecture described herein is therefore best suited for high-accuracy, medium-speed A/D converters in ASIC's. A prototype converter breadboarded using discrete components has confirmed the principles of operation.
\end{abstract}

\section{INTRODUCTION}

A NALOG-TO-DIGITAL (A/D) conversion is an important topic in digital processing of analog signals. Recent work concentrates on the oversampling $\Delta \Sigma$ converter for audio signal processing and the flash converters for video signal processing. In between these two extremes, a successive-approximation method is still widely used for moderate-speed, high-accuracy A/D conversion. In this method, an input analog voltage is converted to the binary number by being compared successively with the quantized threshold voltage sequence which, starting with a half-reference voltage, increases or decreases depending on the bit value determined in the previous cycle to approach the input analog voltage. Therefore, a crucial component in the architecture is a digital-to-analog (D/A) subconverter to produce the threshold voltage sequence.

In bipolar technologies, such a D/A converter is realized by binary-weighted current sources combined with an R-2R ladder. In CMOS technologies, however, it is difficult to implement accurately weighted current sources because of the relatively large variation in threshold voltage of MOS transistors. Thus the binary-weighted capacitor array or its combination with a tapped resistor string is commonly used instead [1], [2]. This solution is straightforward, but requires a large number of unit capacitors. Therefore, a large chip area and a long settling time are inevitable.

Manuscript received July 9, 1992; revised January 25, 1993.

The authors are with the Research Institute of Electronics, Shizuoka University, Hamamatsu 432, Japan.

IEEE Log Number 9209220.
These problems stem from the use of a parallel D/A converter for generating the threshold voltage sequence. A successive-approximation $A / D$ conversion is a serial process. Therefore, replacing the extravagant parallel D/A converter by a simple serial one will resolve the above-mentioned difficulties. Based on this idea, a serial D/A converter is developed which starts a conversion with the most significant bit (MSB) [3], [4]. Following these introductory remarks, the conversion algorithm and the architecture of the successive-approximation A/D converter using this serial $\mathrm{D} / \mathrm{A}$ converter are described. A resolution, a sampling rate, and a power consumption when the A/D converter is fabricated in the monolithic form using present CMOS technologies are estimated by error analyses and Spice simulations. Experimental results obtained by a prototype converter built using discrete components are also given to confirm the principles of operation.

\section{Conversion Algorithm And Architectures}

\section{A. Unipolar Conversion}

Figure 1(a) shows a block diagram of the successiveapproximation A/D converter developed for unipolar conversion [5], [6]. Its operation is controlled by the nonoverlapping three-phase clocks $\phi_{1}, \phi_{2}$, and $\phi_{3}$ and the state signal $S$ which distinguishes between the sample $(S=$ "1") and conversion ( $H=\bar{S}=$ " 1 ") states. Their timing is shown in Fig. 1(b). In the $\phi_{1}=$ " 1 " phase of the sample state, an input analog voltage is sampled into $C_{1}$. In the subsequent conversion state, the analog voltage thus stored in $C_{1}$ is compared with the threshold voltage sequence generated by the D/A converter, to be converted into its digital equivalent.

Now assume that the D/A converter produces the voltage sequence $V_{t}(i)$ in the $i$-th cycle of the conversion state. The voltage $V_{c}(i)$ at the inverting input terminal of the comparator is then given by

$$
\begin{array}{r}
V_{c}(i)=\frac{-C_{1} V_{a}+C_{2} V_{t}(i)}{C_{1}+C_{2}+C_{p}}+V_{\mathrm{os}}, \\
(i=1,2, \cdots, n)
\end{array}
$$

where $C_{p}$ is the parasitic capacitance between the inverting input terminal of the comparator and ground, and $V_{\text {os }}$ is the offset voltage of the comparator. It is clear from (1) 


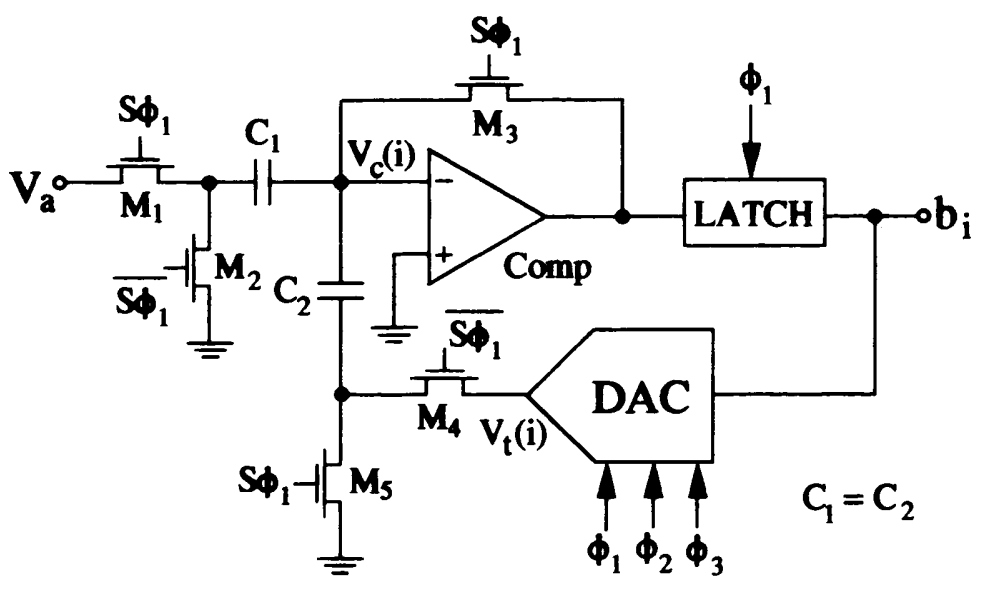

(a)

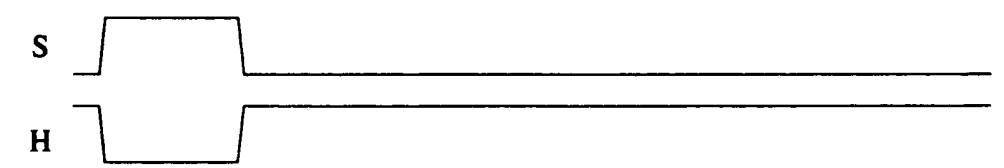

$\mathrm{S}_{1}$
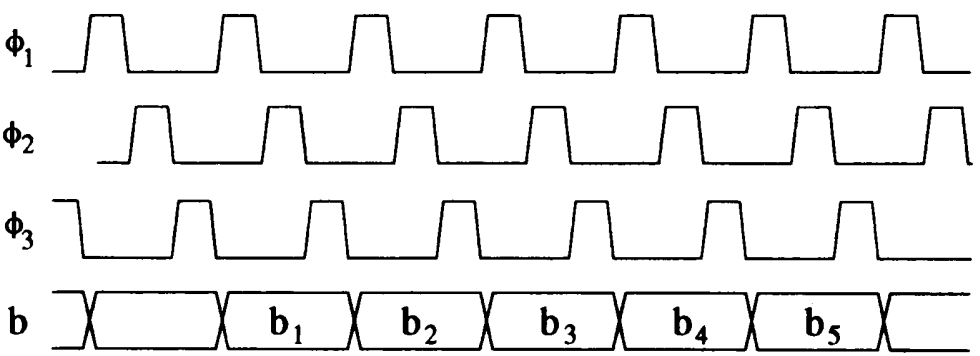

(b)

Fig. 1. A block diagram (a) and timing diagram of digital signals (b) of a successive-approximation $A / D$ converter for unipolar conversion.

that if $C_{1}=C_{2}$, and

$$
V_{t}(i)=\left(\sum_{j=0}^{i-1} b_{j} 2^{-j}+2^{-i}\right) V_{r}
$$

then the value of the $i$-th bit, $b_{i}$, can be determined to be 1 or 0 depending on the output of the comparator. In (2), $V_{r}$ is the reference voltage and $b_{0}=0$. It should also be noted that the conversion process is insensitive to both the parasitic capacitance $C_{p}$ and the offset voltage $V_{\mathrm{os}}$. The capacitance mismatch between $C_{1}$ and $C_{2}$ has no effect upon the conversion linearity, either.

The output $b_{i}$ of the comparator in each cycle indicates the voltage range to which the analog input voltage $V_{a}$ belongs. For instance, assume the values of the first three bits to be $b_{1} b_{2} b_{3}=(101)_{B}$. The most significant bit (MSB) $b_{1}=1$ implies that $V_{a}$ is in the range from $V_{r} / 2$ to $V_{r}$. The next MSB $b_{2}=0$ means that $V_{a}$ is in the range from $V_{r} / 2$ to $(3 / 4) V_{r}$, and $b_{3}=1$ in the range from $(5 / 8) V_{r}$ to $(3 / 4) V_{r}$. It is obvious from this example that the voltage range is successively halved. Let the lower and upper bounds of the voltage range determined in the $i$-th cycle be $V_{L}(i)$ and $V_{U}(i)$, respectively. Then, $V_{L}(i+1)$ and $V_{U}(i+1)$ in the next $(i+1)$ th cycle are given by [4]

$$
\begin{aligned}
V_{L}(i+1) & =V_{L}(i)+b_{i} 2^{-i} V_{r} \\
V_{U}(i+1)-V_{L}(i+1) & =\frac{V_{U}(i)-V_{L}(i)}{2}=2^{-i} V_{r}
\end{aligned}
$$

where $V_{L}(1)=0$ and $V_{U}(1)=V_{r}$. The threshold voltage $V_{t}(i+1)$ required for the next comparison is then given by

$$
V_{t}(i+1)=\frac{V_{L}(i+1)+V_{U}(i+1)}{2} .
$$

Rewriting (2) as

$$
V_{t}(i+1)=\frac{V_{r}}{2}-\sum_{j=1}^{i}(-1)^{b_{j}} 2^{-(j+1)} V_{r}
$$

and expressing (3)-(5) in the recursive forms, we can obtain the algorithm for generating the threshold voltage sequence:

$$
\begin{aligned}
V_{t}(i+1)= & {\left[V_{t}(i)+\overline{b_{i}} V_{L}(i+1)\right.} \\
& \left.+b_{i} V_{U}(i+1)\right] / 2 \\
V_{L}(i+1)= & b_{i} V_{t}(i)+\overline{b_{i}} V_{L}(i) \\
V_{U}(i+1)= & \overline{b_{i}} V_{t}(i)+b_{i} V_{U}(i)
\end{aligned}
$$

where $V_{t}(1)=V_{r} / 2$.

The above recursive algorithms indicate that the D/A converter can be realized by the sample/hold and divideby- 2 stages. Such a realization is shown in Fig. 2 . In the $i$-th cycle of the conversion state, three capacitors $C_{L}, C_{U}$, and $C_{T}$ store the lower bound $V_{L}(i)$, upper bound $V_{U}(i)$, and threshold voltage $V_{t}(i)$ sequences, respectively. $C_{T}$ is a unit capacitor matched to $C_{S}$. In the $\phi_{1}=$ " 1 " phase of the next cycle, either $V_{L}(i)$ or $V_{U}(i)$ is updated to $V_{t}(i)$, depending on the bit value $b_{i}$ determined by the previous conversion. In the next $\phi_{2}=$ " 1 " phase, $C_{T}$ is charged to the new $V_{L}(i+1)$ or $V_{U}(i+1)$. In the subsequent $\phi_{3}$ $=$ " 1 " phase, $C_{T}$ and $C_{S}$ are connected in parallel to perform the divide-by-2 operation involved in (7). The threshold voltage sequence $V_{t}(i+1)$ thus generated is compared with the input analog voltage, to determine the value of the next bit $b_{i+1}$. The operation of the D/A converter is also insensitive to parasitic capacitances and offset voltages of op-amps.

\section{B. Bipolar Conversion}

In the unipolar conversion described above, the analog input voltage $V_{a}$ with positive polarity is compared with the positive reference voltage $V_{r}$ by subtracting $V_{r}$ from $V_{a}$. The subtraction is replaced by the addition, in fact, by inverting the polarity of $V_{a}$ stored in $C_{1}$. Therefore, the architectures in the previous section can be extended to a bipolar conversion if they are modified such that the D/A converter produces the negative threshold voltage sequence when an input analog voltage is negative. Such modifications are shown in Figs. 3 and 4.

An extra state, designated by $H_{1}$, is introduced into the timing diagram shown in Fig. 3(b) to detect the polarity of an input analog voltage $V_{a}$. The sign bit $b_{0}$ is set to 1 if $V_{a} \geq 0$, or to 0 otherwise. This value is held during the subsequent conversion state $\mathrm{H}_{2}$ by the latch 2 shown in Fig. 3(a). It should be pointed out here that because of 


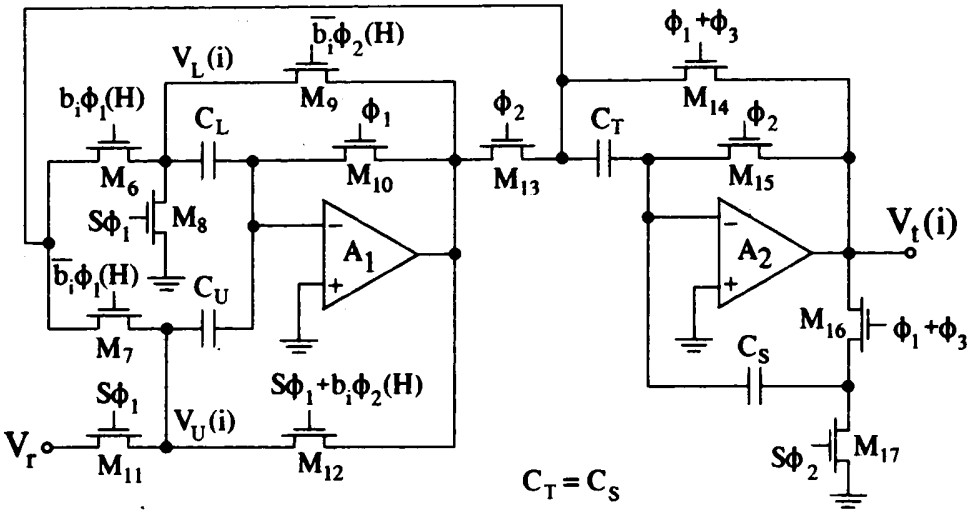

Fig. 2. A circuit diagram of the D/A converter for unipolar conversion.

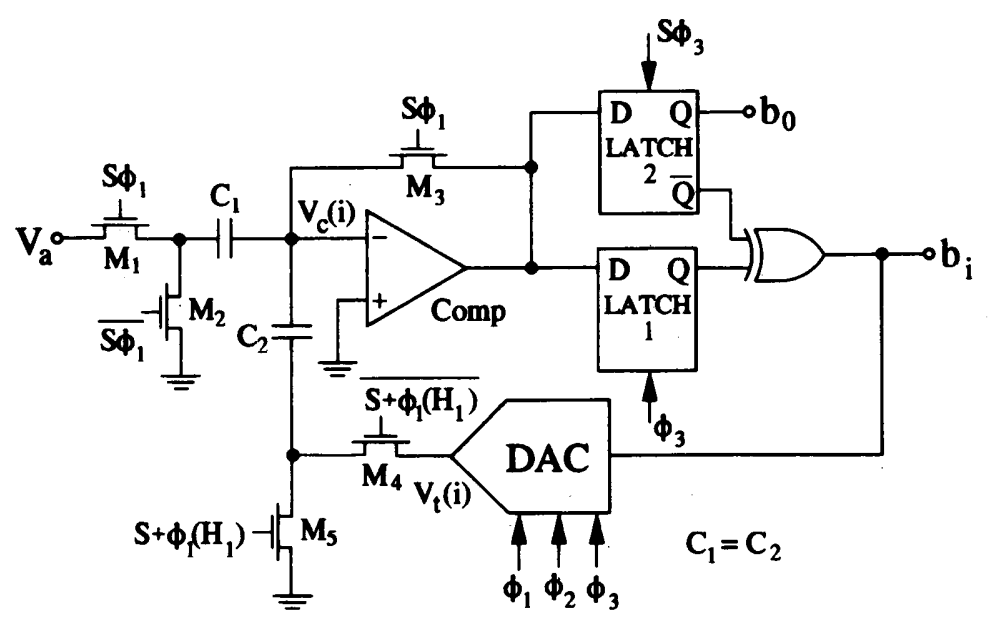

(a)

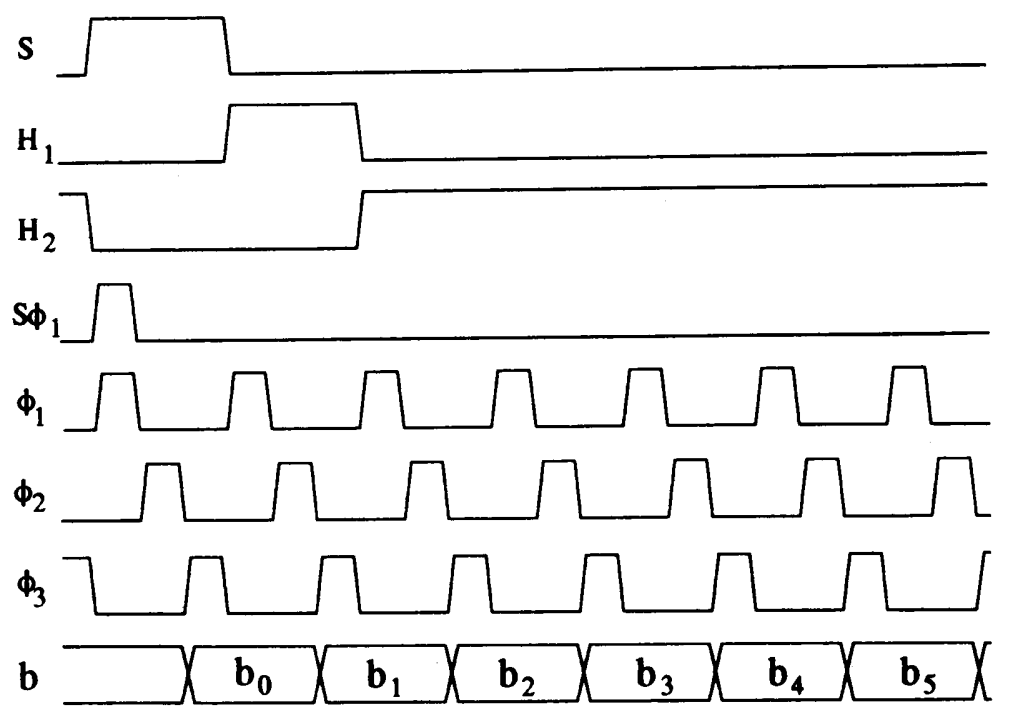

(b)

Fig. 3. A block diagram (a) and timing diagram of digital signals (b) of a successive-approximation $A / D$ converter for bipolar conversion.

the polarity inversion, the magnitude relation between $V_{a}$ and $V_{t}(i)$ is reversed when $V_{a}$ is negative. The ExclusiveOR gate is used in Fig. 3(a) to retrieve the correct relation.

The recursive algorithms for generating the threshold voltage sequence are the same as those in the unipolar conversion, except for $V_{r}$ being replaced by $-(-1)^{b_{0}} V_{r}$. The D/A converter modified is shown in Fig. 4. It produces the bipolar threshold voltage sequence as follows. In the $\phi_{1}=$ " 1 "' phase of the sample state, matched ca-

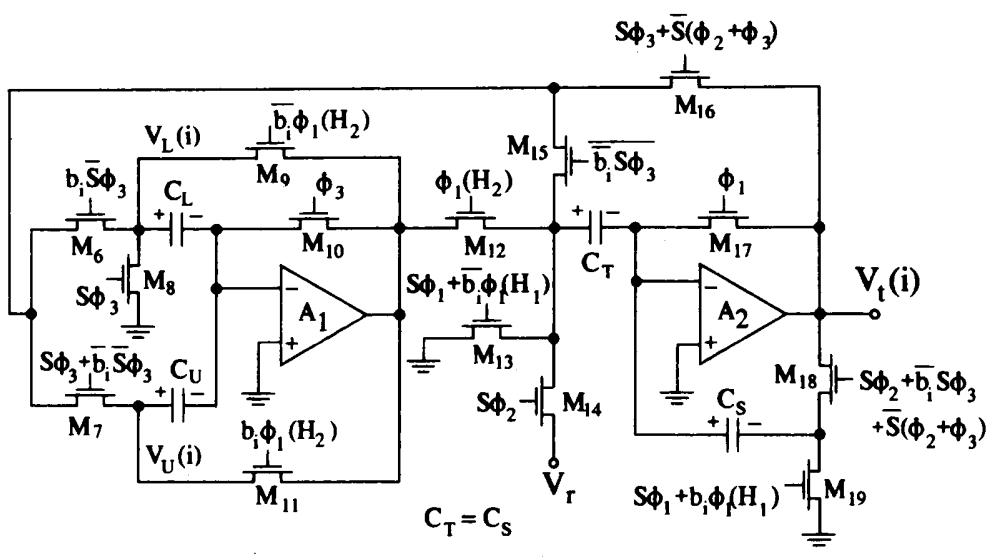

Fig. 4. A circuit diagram of the $\mathrm{D} / \mathrm{A}$ converter for bipolar conversion.

pacitors $C_{T}$ and $C_{S}$ are both discharged. Then in the next $\phi_{2}=$ "1" phase, $C_{T}$ and $C_{S}$ are charged to $V_{r}$ with the polarity indicated. In the next $\phi_{3}=$ " 1 " phase, the value of $b_{0}$ is determined. If $b_{0}=1$, op-amp $A_{2}$ holds the positive reference voltage stored in $C_{T}$, to charge $C_{U}$ to $V_{r}$, while op-amp $A_{1}$ discharges $C_{L}$ to ground. Therefore, the lower and upper bounds start with $V_{L}(0)=0$ and $V_{U}(0)$ $=V_{r}$, respectively. The charge stored in $C_{S}$ is discharged to ground in the $\phi_{1}=$ " 1 " " phase of the $H_{1}$ state. If $b_{0}=$ 0 , on the other hand, op-amp $A_{2}$ holds the negative reference voltage stored in $C_{T}$, to charge $C_{U}$ to $-V_{r}$. Therefore, the upper bounds start with $V_{U}(0)=-V_{r}$. In either case, the D/A converter follows the same process as that of the unipolar conversion in the subsequent conversion state $\left(H_{2}=\right.$ " 1 "'), to generate the threshold voltage sequence. The operation is insensitive again to parasitic capacitances and the offset voltages of op-amps.

\section{CMOS DESIGN}

The op-amp and the latched comparator have been designed in CMOS. Figure 5 shows a circuit diagram of the op-amp. The $\mathrm{W} / \mathrm{L}$ ratios, assuming a $3-\mu \mathrm{m}$ process, are also indicated. The circuitry consists of the input differential pair $M_{1}$ and $M_{2}$, folded cascade stage $M_{7}$ and $M_{8}$, output stage $M_{3}-M_{6}$, constant current source $M_{9}$, current mirror $M_{10}$ and $M_{11}$, and bias chain $M_{12}-M_{15}$ [7]-[9]. The folded cascade stage makes it possible to realize a high gain without causing the instability. Figure 6(a) shows the frequency characteristics of the op-amp. It is obvious that the load capacitance $C_{L}$ and the output impedance form the dominant pole. The second pole due to the stray capacitances at the low-impedance node is estimated from the phase characteristics to be at about $500 \mathrm{MHz}$. These pole locations lead to the unity-gain frequency extending beyond $100 \mathrm{MHz}$. Connecting the source follower to lower the output impedance, one can obtain the wider bandwidth. Figure 6(b) shows the transient response of the op-amp simulated using Spice. The output voltage of the op-amp loaded by a 2-pF capacitor settles within $0.1 \%$ of the final value in $80 \mathrm{~ns}$. The $0.1 \%$ settling time can be reduced to $40 \mathrm{~ns}$ if the load is decreased to $1 \mathrm{pF}$.

Figure 7 shows a circuit diagram of the latched comparator. The comparator section consists of four cascaded 


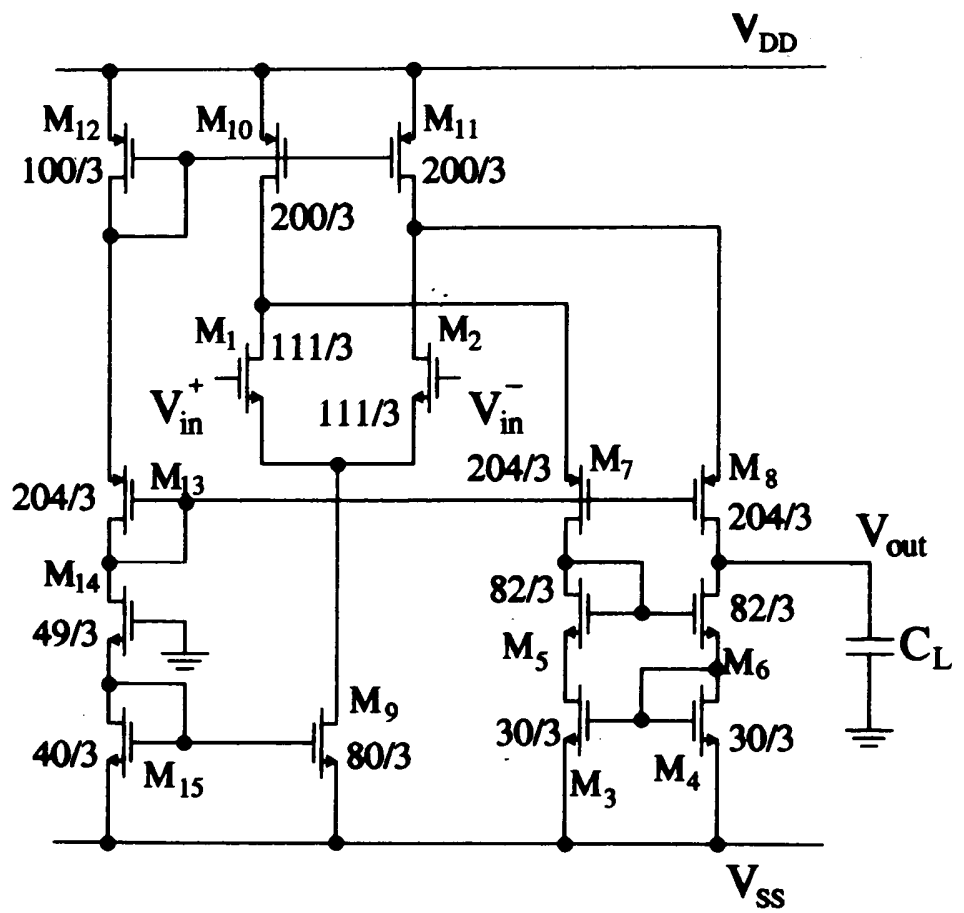

Fig. 5. A circuit diagram of the op-amp.

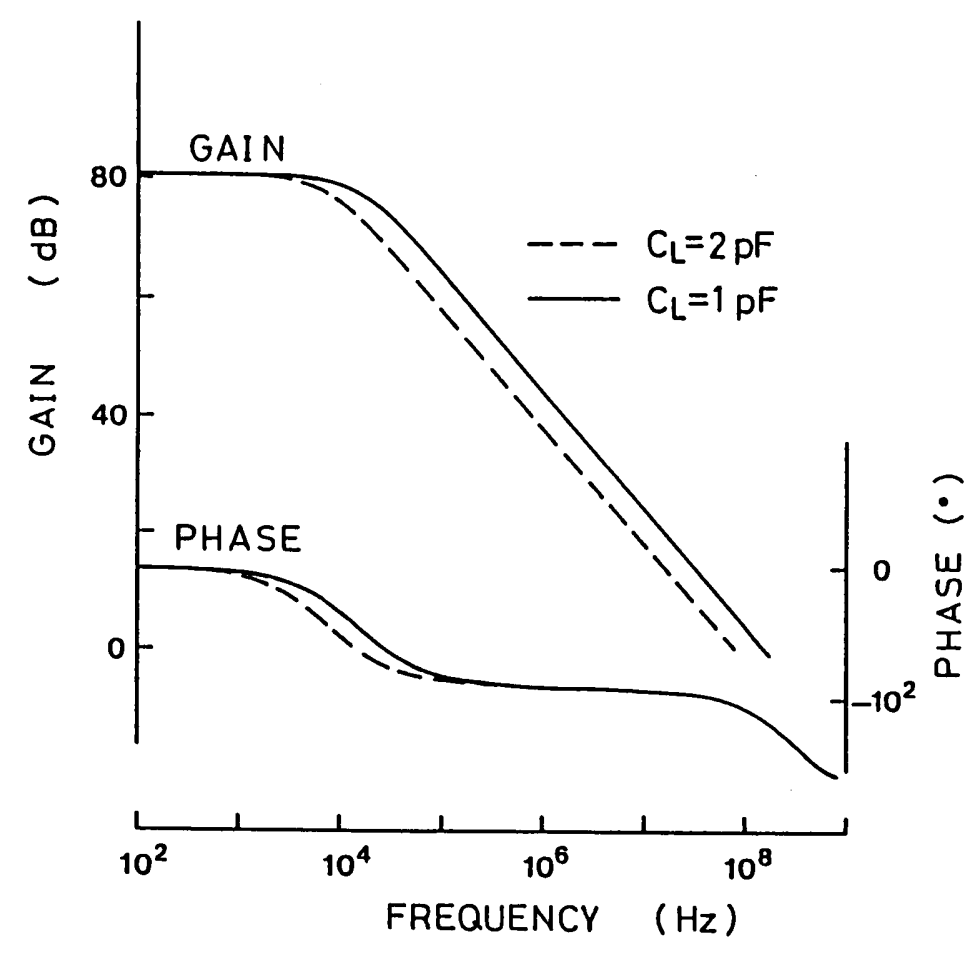

(a)

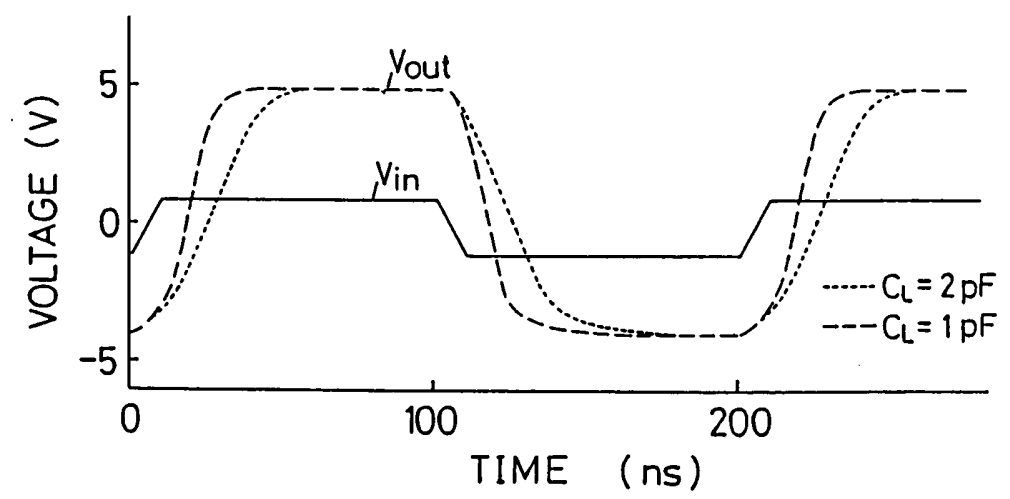

(b)

Fig. 6. The frequency characteristics (a) and the transient response (b) of the op-amp.

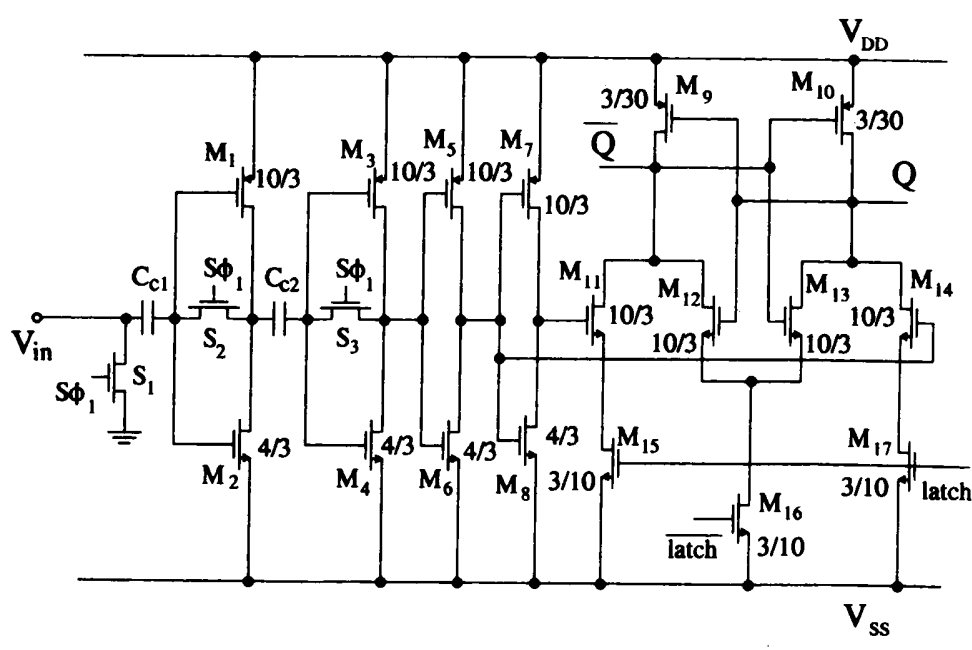

Fig. 7. A circuit diagram of the latched comparator.

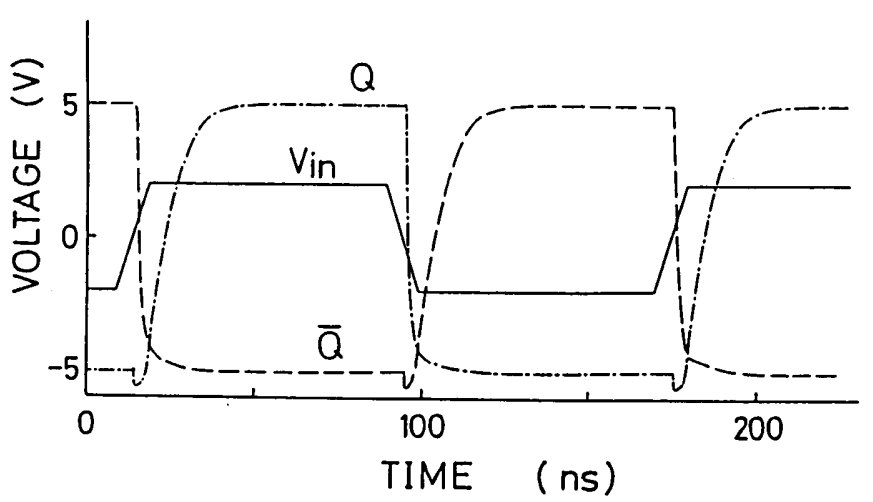

Fig. 8. The transient response of the latched comparator.

inverter stages. Capacitors $C_{c 1}$ and $C_{c 2}$ are used for autozeroing. The charge injection due to switches $S_{2}$ and $S_{3}$ is reduced to negligibly small by arranging the switching sequence [10]. This section is followed by the latch composed of four cross-coupled inverter stages. According to Spice simulations, the input offset voltage of the comparator is $0.2 \mathrm{mV}$ when $V_{D D}=-V_{S s}=5 \mathrm{~V}$. Figure 8 shows the transient response. The response time including the delay in the latch is $30 \mathrm{~ns}$.

\section{Performance Estimates}

Based on the simulation results presented in the previous section, the performance of the successive-approximation $A / D$ converter when implemented using a 3- $\mu \mathrm{m}$ CMOS process is estimated. The estimation assumes a unipolar conversion, but the results are applied well to a bipolar conversion because of almost the same circuit configuration.

\section{A. Accuracy}

As described in Section II, parasitic capacitances and offset voltages of op-amps and a comparator have no effect on the conversion process. Capacitance mismatch between $C_{1}$ and $C_{2}$ causes a gain or scale error, but this can be compensated by adjusting the reference voltage. Therefore, the main error sources involved in the present architecture are the finite gain of op-amps, charge injec- 
tion due to clock feedthrough, and capacitance mismatch between $C_{T}$ and $C_{S}$.

Let the open-loop gain of op-amps $A_{1}$ and $A_{2}$ be $A$. Then the recursive form (7) of the threshold voltage sequence is modified as follows:

$$
\begin{aligned}
V_{t}^{\prime}(i+1)= & {\left[\frac{A}{A+1}\right] \cdot \frac{V_{t}^{\prime}(i)}{2}+\left[\frac{A}{A+1}\right]^{2} } \\
& \cdot \frac{b_{i} V_{U}(i+1)+\bar{b}_{i} V_{L}(i+1)}{2}
\end{aligned}
$$

where $V_{t}^{\prime}(1)=[A /(A+1)] \cdot\left(V_{r} / 2\right)$, and a prime denotes the erroneous quantity. This erroneous sequence causes the nonlinearity which depends on the bit pattern. Figures 9(a) and (b) show the differential (DNL) and integral nonlinearity (INL) in 11-b A/D conversion when $A$ is $80 \mathrm{~dB}$. These simulations indicate that the finite gain affects the INL more seriously than the DNL, and that INL becomes maximum when all bits assume 0 . If the maximum error is less than $1 \mathrm{LSB}$, then the conversion is accurate down to its LSB. Formulating this condition, we have the criterion:

$$
2^{n}<\frac{2\left(A^{2}+3 A+2\right)}{A^{2}\left[\frac{A}{2(A+1)}\right]^{n}+3 A+2} .
$$

Now assume that switches $M_{10}$ and $M_{15}$ in Fig. 2 inject the feedthrough charge $Q_{f}$ into the inverting input terminals of op-amps $A_{1}$ and $A_{2}$, respectively, when they turn off. The threshold voltage sequence including $Q_{f}$ is given by

$$
\begin{aligned}
V_{t}^{\prime}(i+1)= & \frac{V_{t}^{\prime}(i)+b_{i} V_{U}(i+1)+\bar{b}_{i} V_{L}(i+1)}{2} \\
& +\frac{Q_{f}}{C_{n}}
\end{aligned}
$$

where $V_{t}^{\prime}(1)=V_{r} / 2+Q_{f} / C_{n}$ and $C_{n}=C_{S}=C_{T}$. It is found by the simulations based on (12) that the DNL and INL are of almost the same order of magnitude and independent of the bit pattern. The condition to achieve the 1 LSB accuracy is given by

$$
2^{n}<1+\frac{C_{n} V_{r}}{2 Q_{f}}
$$

The threshold voltage sequence when capacitor mismatch $\epsilon$ is present between $C_{T}$ and $C_{S}$ is given by

$$
\begin{aligned}
V_{t}^{\prime}(i+1) \\
\quad=\frac{(1-\epsilon) V_{t}^{\prime}(i)+b_{i} V_{U}(i+1)+\overline{b_{i}} V_{L}(i+1)}{2-\epsilon}
\end{aligned}
$$

where $V_{t}^{\prime}(1)=[(1-\epsilon) /(2-\epsilon)] \cdot V_{r}$. Simulations based on (14) indicate that capacitance mismatch degrades se-

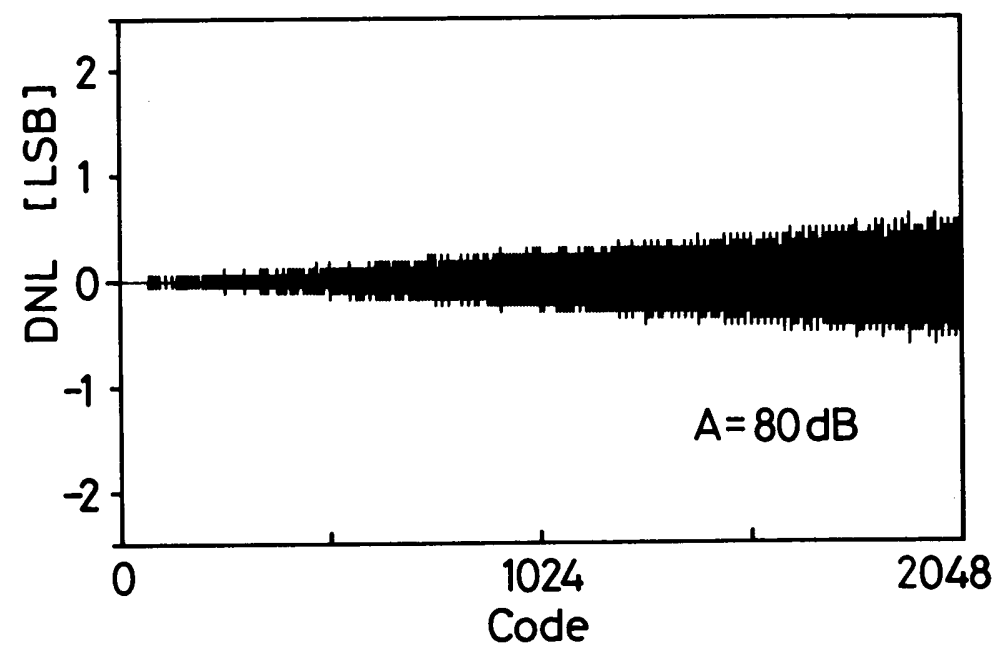

(a)

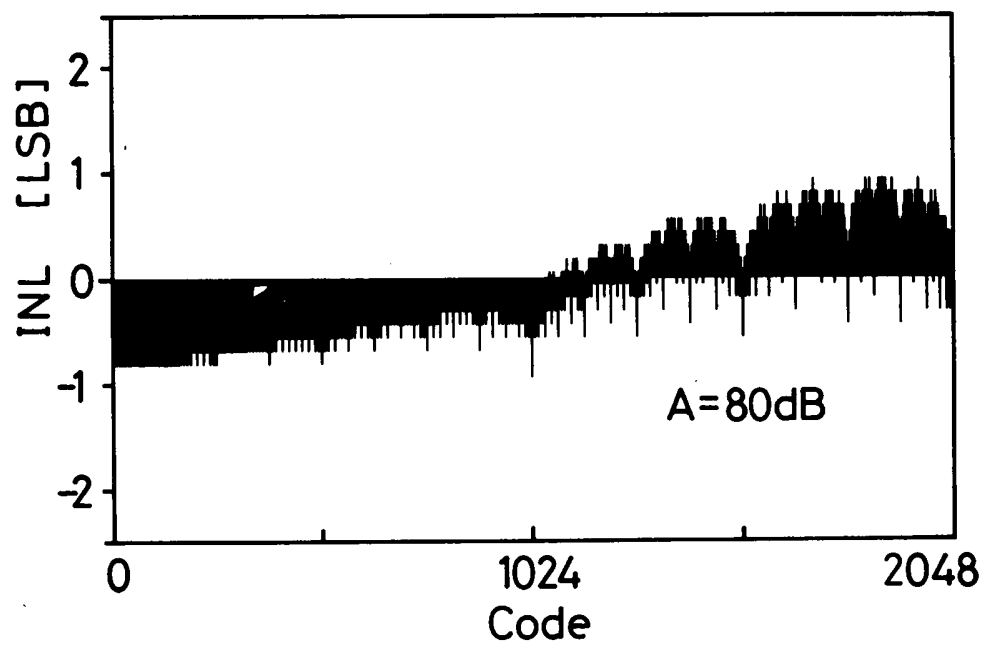

(b)

Fig. 9. The differential nonlinearity (DNL) (a) and the integral nonlinearity (INL) (b) in 11-bit A/D conversion when the finite gain of op-amps is $80 \mathrm{~dB}$.

riously the INL. The condition for the maximum INL to be less than 1 LSB in an $n$-bit conversion is given by

$$
\epsilon<\frac{2}{2^{n-2}+1} \text {. }
$$

From (11), (13), and (15), one can obtain the requirements for, say, 11-b resolution as follows: $A>80 \mathrm{~dB}$, $Q_{f} / C_{n}<0.5 \mathrm{mV}$ when $V_{r}=2 \mathrm{~V}$, and $\epsilon<0.25 \%$. The latter two requirements are easily met by present MOS technologies. Therefore, the op-amp design is the most critical in realizing a high resolution. The gain-compensated scheme proposed by Nagaraj $e t$ al. is effective to relax the gain requirement [11], [12]. The D/A converter accommodating such a scheme is shown in Fig. 10. Driven by the four-phase clock, the circuit compensates for the gain error based on the following principle. The gain error term $A /(A+1)$ in (10) is approximated, to first order, by $1-1 / A$. The error voltage due to the finite gain is thus $-V_{o} / A$, where $V_{\iota}$ is $V_{U}(i)$ or $V_{L}(i)$ for opamp $A_{1}$ and $V_{t}(i)$ for op-amp $A_{2}$. Op-amp $A_{1}$ detects the error voltage and stores it into $C_{I}$ in the $\phi_{1}$ and $\phi_{2}$ phases. The error voltage stored in $C_{I}$ cancels the gain error that 


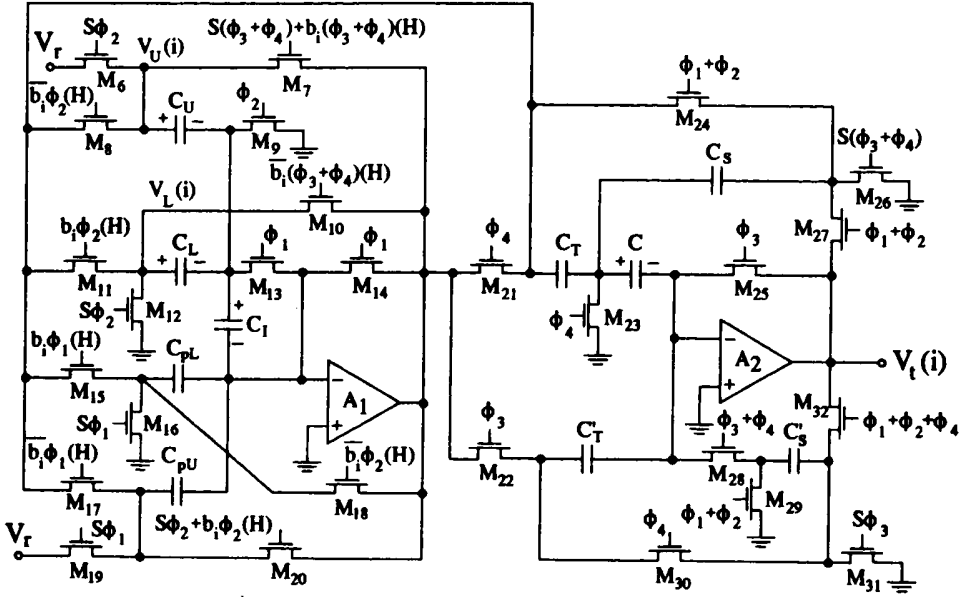

Fig. 10. A circuit diagram of the gain-compensated D/A converter.

would otherwise appear in the $\phi_{3}$ and $\phi_{4}$ phases. Though the clock phases differ, op-amp $A_{2}$ operates similarly to cancel the first-order gain error. The residual error is therefore of the second order, which implies that the effective gain is $A^{2}$. Referring to Fig. 6(a), the open-loop gain of the aforementioned op-amp is $42 \mathrm{~dB}$ at $1 \mathrm{MHz}$ when the load is $1 \mathrm{pF}$. A resolution higher than 11-b can be expected from the improved D/A architecture, if the clock is lower than $1 \mathrm{MHz}$. Since the open-loop gain rolls off at $-6 \mathrm{~dB} /$ oct, the resolution decreases to $9-\mathrm{b}$ at 5 $\mathrm{MHz}$.

\section{B. Conversion Speed}

The duration of each phase necessary for the proper operation can be determined by examining the operation of the D/A converter shown in Fig. 10. In the $\phi_{1}$ phase, $C_{T}$ and $C_{S}$ share the charge, and op-amp $A_{2}$ outputs the new $V_{t}(i)$ thus produced, to determine the value of $b_{i}$. Assume that all the capacitors are $C=1 \mathrm{pF}$, and the 'on' resistance $R_{\mathrm{on}}$ of each switch is $2 \mathrm{k} \Omega$. Then, the $\phi_{1}$ phase should last $t_{s}+7 \mathrm{CR}_{\text {on }}+t_{r}=84 \mathrm{~ns}$, where $t_{s}=40 \mathrm{~ns}$ is the $0.1 \%$ settling time of the op-amp and $t_{r}=30 \mathrm{~ns}$ is the response time of the latched comparator. The charging time of $C_{p U}$ or $C_{p L}$ is $14 \mathrm{CR}_{\mathrm{on}}=28 \mathrm{~ns}$, which is shorter than $t_{r}$. In the $\phi_{2}$ phase, op-amp $A_{2}$ charges $C_{U}$ or $C_{L}$ to $V_{t}(i)$, while op-amp $A_{1}$ holds the voltage across $C_{p U}$ or $C_{p L}$. The time required for charging $C_{U}$ or $C_{L}$ is $28 \mathrm{~ns}$, which is shorter than the $40 \mathrm{~ns}$ required for op-amp $A_{1}$ to settle. The time required for the $\phi_{3}$ phase can be calculated by the same procedure to be $t_{s}+14 \mathrm{CR}_{\mathrm{on}}=68 \mathrm{~ns}$, and that for the $\phi_{4}$ phase to be $t_{s}+7 \mathrm{CR}_{\mathrm{on}}=54 \mathrm{~ns}$. Some of the above processes are carried out concurrently. Therefore, the time required for a 1-b conversion is estimated to be $230 \mathrm{~ns}$. This results in the maximum sampling rate $440 \mathrm{ksps}$ with 10 -b resolution.

\section{Power Consumption}

The op-amp shown in Fig. 5, which is designed to slew at $280 \mathrm{~V} / \mu \mathrm{s}$, dissipates $14 \mathrm{~mW}$ when $\mathrm{V}_{\mathrm{DD}}=-\mathrm{V}_{\mathrm{SS}}=5$ $\mathrm{V}$ and $C_{L}=2 \mathrm{pF}$. The power dissipation of the latched comparator is $16 \mathrm{~mW}$. The total power consumption, in-

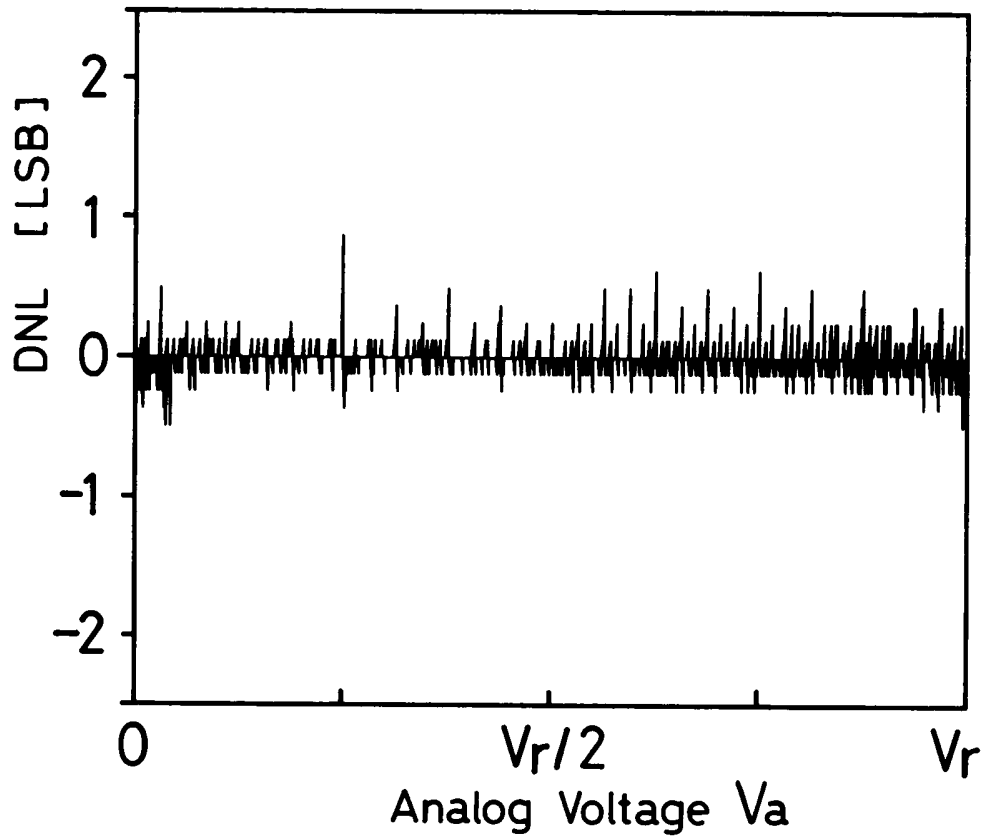

(a)

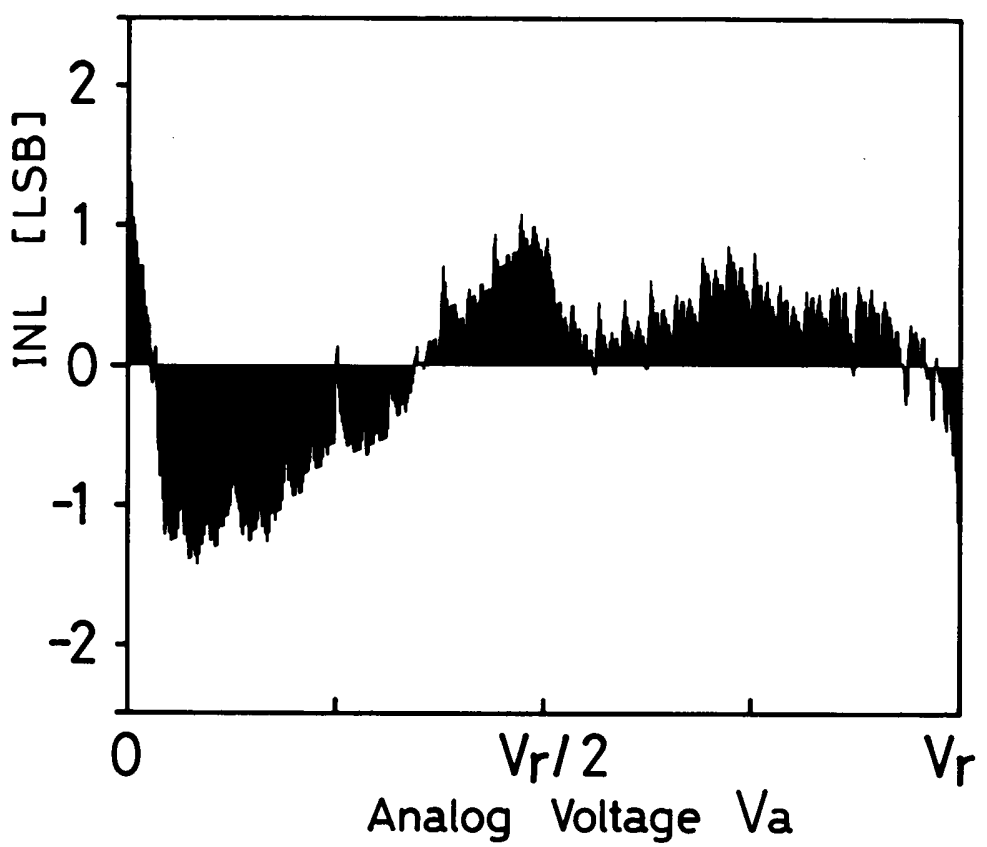

(b)

Fig. 11. The differential nonlinearity (DNL) (a) and the integrated nonlinearity (INL) (b) of the prototype 9 -bit successive-approximation A/D converter.

cluding the power dissipated in the digital circuitry and the reference source, is estimated to be less than $60 \mathrm{~mW}$.

\section{Prototype Converter}

To confirm the principles of operation, a prototype converter based on Figs. 1 and 2 was breadboarded using discrete components. Op-amps and switches used are LF356 and MC14016, respectively. All the capacitors are 5.6 $\mathrm{nF}$. Capacitance mismatch between $C_{T}$ and $C_{S}$ is less than $0.5 \%$. The three-phase clock frequency is $20 \mathrm{kHz}$.

The DNL and INL of the 9-b prototype converter measured by a dc test are shown in Figs. 11(a) and (b), respectively. The maximum DNL is $0.875 \mathrm{LSB}$, and thus no missing code appears in the transfer characteristics. The maximum INL is $1.425 \mathrm{LSB}$. The open-loop gain of 
the op-amps used is $50 \mathrm{~dB}$ at $20 \mathrm{kHz}$. The accuracy calculated by substituting this value for $A$ in (11) agrees well with the measurement result.

\section{Conclusions}

A switched-capacitor successive-approximation A/D converter has been described which incorporates a serial D/A subconverter for generating the threshold voltage sequence. Circuit diagrams for the CMOS implementation were also described. Error analyses and Spice simulations show that a resolution higher than $11 \mathrm{~b}$, a sampling rate up to $440 \mathrm{ksps}$ with 10 -b resolution, and a power consumption less than $60 \mathrm{~mW}$ can be expected from its monolithic realization using present 3- $\mu \mathrm{m}$ CMOS technologies. A higher sampling rate is possible with the time-multiplexed architecture [13], or with finer linewidths. It also features a low device count integrable onto a small chip area. These distinctive features make the present architecture most suited for analog interfaces of mixed analog and digital ASIC's.

\section{REFERENCES}

[1] J. L. McCreary and P. R. Gray, "All-MOS charge redistribution analog-to-digital conversion techniques-Part I," IEEE J. Solid-State Circuits, vol. SC-10, pp. 371-379, Dec. 1975.
[2] B. Fotouhi and D. A. Hodges, "High-resolution A/D conversion in MOS/LSI," IEEE J. Solid-State Circuits, vol. SC-14, pp. 920-926, Dec. 1979.

[3] K. Watanabe, G. C. Temes, and Y. Moriuchi, "A new digital-toanalog conversion algorithm," in Proc. IEEE Int. Symp. Circuits and Systems, 1988, pp. 2817-2820.

[4] K. Watanabe, G. C. Temes, and T. Tagami, "A new algorithm for cyclic and pipeline data conversion," IEEE Trans. Circuits and Systems, vol. 37, pp. 249-252, Feb. 1990.

[5] D. K. Shum and G. C. Temes, "Stray-insensitive differential input stages for A/D converters," Electron. Lett., vol. 20, pp. 1041-1042, Dec. 1984.

[6] S. Ogawa and K. Watanabe, "A switched-capacitor successive-approximation analog-to-digital converter," in Proc. IEEE Int. Symp. Circuits and Systems, 1991, pp. 1577-1580.

[7] R. Gregorian and G. C. Temes, Analog MOS Integrated Circuits for Signal Processing. New York: Wiley, 1986, chap. 4.

[8] P. R. Gray and R. G. Meyer, Analysis and Design of Analog Integrated Circuits. New York: Wiley, 1984, chap. 12.

[9] P. R. Gray and R. G. Meyer, "MOS operational amplifier designA tutorial overview," IEEE J. Solid-State Circuits, vol. SC-17, pp. 969-982, Dec. 1982.

[10] D. J. Allstot, "A precision variable-supply CMOS comparator," IEEE J. Solid-State Circuits, vol. SC-17, pp. 1080-1087, Dec. 1982.

[11] K. Nagaraj, K. Singhal, T. R. Viswanathan, and J. Vlach, "Reduction of finite-gain effect in switched-capacitor filters," Electron. Lett., vol. 21, pp. 644-645, July 1985.

[12] K. Nagaraj, T. R. Viswanathan, K. Signhal, and J. Vlach, "Switchedcapactor circuits with reduced sensitivity to amplifier gain," IEEE Trans. Circuits and Systems, vol. CAS-34, pp. 571-574, May 1987.

[13] W. C. Black and D. A. Hodges, "Time interleaved converter arrays," IEEE Trans. Circuits and Systems, vol. SC-15, pp. 1022-1029, Dec. 1980. 